\title{
Effective field theory and scalar extensions of the top quark sector
}

\author{
Christoph Englert $\odot,{ }^{1, *}$ Peter Galler $\odot,{ }^{1, \dagger}$ and Chris D. White ${ }^{2, \$}$ \\ ${ }^{1}$ SUPA, School of Physics and Astronomy, University of Glasgow, Glasgow G12 8QQ, United Kingdom \\ ${ }^{2}$ Centre for Research in String Theory, School of Physics and Astronomy, \\ Queen Mary University of London, 327 Mile End Road, London E1 4NS, United Kingdom
}

(Received 26 August 2019; accepted 30 January 2020; published 27 February 2020)

\begin{abstract}
Effective field theory (EFT) approaches are widely used at the Large Hadron Collider (LHC), such that it is important to study their validity and ease of matching to specific new physics models. In this paper, we consider an extension of the Standard Model (SM) in which a top quark couples to a new heavy scalar. We find the dimension six operators generated by this theory at low energy and match the EFT to the full theory up to the next-to-leading order (NLO) precision in the simplified model coupling. We then examine the range of validity of the EFT description in top pair production, finding excellent validity even if the scalar mass is only slightly above LHC energies, provided NLO corrections are included. In the absence of the latter, the LO EFT overestimates kinematic distributions, such that overoptimistic constraints on beyond the Standard Model (BSM) contributions are obtained. We next examine the constraints on the EFT and full models that are expected to be obtained from both top pair and four top production at the LHC, finding for low scalar masses that both processes show similar exclusion power. However, for larger masses, estimated LHC uncertainties push constraints into the nonperturbative regime, where the full model is difficult to analyze, and thus is not perturbatively matchable to the EFT. This highlights the necessity to improve uncertainties of SM hypotheses in top final states.
\end{abstract}

DOI: 10.1103/PhysRevD.101.035035

\section{INTRODUCTION}

The potential discovery of new physics beyond the Standard Model (BSM) remains one of the principal motivations of contemporary high energy physics research, in both theory and experiment. Much attention focuses on the top quark and its antiparticle, given that these are the heaviest particles in the SM, whose behavior is thus likely to be particularly sensitive to BSM effects. Furthermore, they are of fundamental importance when discussing the naturalness (or otherwise) of the electroweak symmetry breaking scale, such that typical BSM scenarios necessarily involve modifications of the top sector. Their effects may then be easier to investigate experimentally than purely electroweak processes (e.g., Higgs production), owing to the relatively large production cross sections of top particles at the Large Hadron Collider (LHC) (see e.g., Ref. [1] for a recent review).

\footnotetext{
*christoph.englert@glasgow.ac.uk

†peter.galler@glasgow.ac.uk

*christopher.white@qmul.ac.uk
}

Published by the American Physical Society under the terms of the Creative Commons Attribution 4.0 International license. Further distribution of this work must maintain attribution to the author(s) and the published article's title, journal citation, and DOI. Funded by SCOAP.
As is well-known there are, broadly speaking, two main ways to investigate possible new physics. The first is to assume a particular BSM theory, and to look for associated signatures, such as the resonant production of new particles e.g., decaying to a top pair. This is necessarily highly model-dependent, and the lack of convincing new physics signatures at the LHC to date instead motivates the use of model-independent approaches. Chief among these is perhaps effective field theory (EFT), in which one considers the SM Lagrangian to be the leading term in an expansion in gauge-invariant higher-dimensional operators. One may then extend the SM Lagrangian by these higher dimensional terms, where dimension six is the state of the art [2-8] (for a review see [9]). Each correction to the SM is suppressed by one or more inverse powers of the new physics scale, and thus such a framework is applicable only if this scale (e.g., a new particle mass) exceeds the typical energy scales that are probed in a particular collider of interest.

An intermediate approach between EFT and concrete UV scenarios is represented by so-called simplified models (for reviews see [10-12]), which aim to reproduce a broad class of kinematic properties of the full UV theories parametrized by only a few additional propagating degrees of freedom and their couplings. The relation to the EFT approach is that certain classes of operators have then been resummed to arbitrarily high mass dimension, and certain 
extensions of the SM might be particularly generic or wellmotivated, e.g., Higgs mixing models. Furthermore, experimental collaborations will in any case investigate large classes of simplified models. It is then instructive, for particular examples, to compare the two techniques, particularly with regard to how constraints from the approaches to new physics compare with each other in different kinematic regions.

In this paper, we perform a case study of this idea in the top quark sector, which has been the subject of a number of recent EFT studies [13-42]. We will consider a particular model, in which the SM is supplemented by an additional scalar, whose behavior is parametrized entirely by its mass and couplings. We will calculate the top pair production cross section including the effects of this new particle up to one-loop order, showing explicitly which dimension six SM EFT operators are generated upon taking the mass to be asymptotically large. Matching of the EFT (see also [43-49] for generic approaches) to the full theory can be performed at (next-to-)leading order $[(\mathrm{N}) \mathrm{LO}]$ in the coupling space of the latter, so that we have potentially three different descriptions of the new physics: (i) the LO EFT description; (ii) the NLO EFT description, in which more effective operators are generated; (iii) the full simplified model. We can then examine the validity of each approach and the ease of matching EFT constraints to the full theory.

We will first focus on top quark pair production, demonstrating explicitly that an EFT description can provide an excellent approximation to the full model, as expected. However, we will see that NLO corrections in the EFT approach are particularly important, and that a naïve LO approach tends to overestimate kinematic distributions, such that its (invalid) application would lead to overoptimistic constraints on new physics.

The operators examined in this paper also affect four top production [26,50,51], which is actively being searched for by both the ATLAS [52] and CMS [53,54] Collaborations. We examine the projected constraints on this process (and top pair production) that are expected to be obtained after the high luminosity LHC (HL-LHC) upgrade [55-57], and convert these into constraints on the parameter space of the new physics model. We will see that constraints from four top production are competitive with top pair production, suggesting that the two processes would have roughly comparable weights in a global EFT fit. However, the extrapolated uncertainties from both top pair and four top production lead to constraints that probe parameter space regions in which the full theory is nonperturbative. For large scalar masses, the width of the scalar resonance increases, such that no meaningful constraint on the coupling is obtained in the full theory. Thus, while constraints in the EFT description remain in principle valid and are possible, it becomes impossible to match the EFT description to the full theory of new physics, given that perturbative computations in the latter are not obtainable.
The model considered here has been widely studied in a number of different new physics scenarios. Thus, we hope that our results provide a useful case study for the application of EFT at the LHC, which will inform pragmatic discussions about how to apply this technique going forwards, and what can be learned (or otherwise) about specific UV completions. The structure of our paper is as follows. In Sec. II we introduce the simplified model (of an additional scalar particle) that we are considering, and calculate the corrections to top pair production up to NLO. We furthermore explain how the EFT description is obtained at low energy (relative to the scalar mass). In Sec. III, we present numerical results for the top invariant mass distribution, and demonstrate the validity of the EFT description, even at LO, when the scalar mass is asymptotically large. We then quantify the mass regime in which the NLO-matched EFT description is a good approximation of the full theory. In Sec. IV, we examine the projected uncertainties on top pair and four top production at the LHC, and we examine the constraints obtained in the EFT at (N)LO, as well as the full theory. Finally, in Sec. V, we discuss our results and conclude.

\section{A SIMPLIFIED MODEL AND ITS EFT LIMIT}

In this work, we consider a simplified model (similar to Ref. [58]) with dominant couplings to the top quark

$$
\mathcal{L}_{\mathrm{BSM}}=\frac{1}{2} \partial_{\mu} S \partial^{\mu} S-\frac{1}{2} m_{S}^{2} S^{2}-\left(c_{S} \bar{t}_{L} t_{R} S+\text { H.c. }\right),
$$

where $S$ is a scalar field of mass $m_{S}{ }^{1}$ Provided the latter is greater than $2 m_{t}$, where $m_{t}$ is the top mass, the scalar $S$ may directly decay into (anti)top pairs, with corresponding width

$$
\Gamma(S \rightarrow t \bar{t})=\frac{3 c_{S}^{2} m_{S}}{8 \pi} \sqrt{1-\frac{4 m_{t}^{2}}{m_{S}^{2}}} \equiv c_{S}^{2} \tilde{\Gamma} .
$$

Further contributions to the width arise from the fact that $S$ can couple to gluons and photons via a top quark loop, analogously to the SM Higgs boson. Although we include the loop-induced decays for completeness, the prompt decay $S \rightarrow t \bar{t}$ dominates over the entire considered mass range.

Our aim in this paper is to compare an EFT description of the theory of Eq. (1) at low energy, with the full theory, in order to assess the validity and interpretation of the former. To this end, let us consider how this theory leads to corrections to top pair production up to NLO in the coupling of the scalar, i.e., up to and including $\mathcal{O}\left(c_{S}^{2}\right)$. Comparison with the EFT will then allow us to match the two descriptions. Representative diagrams contributing to

\footnotetext{
${ }^{1}$ Similar frameworks have been considered in flavour changing neutral current studies, e.g., [59].
} 

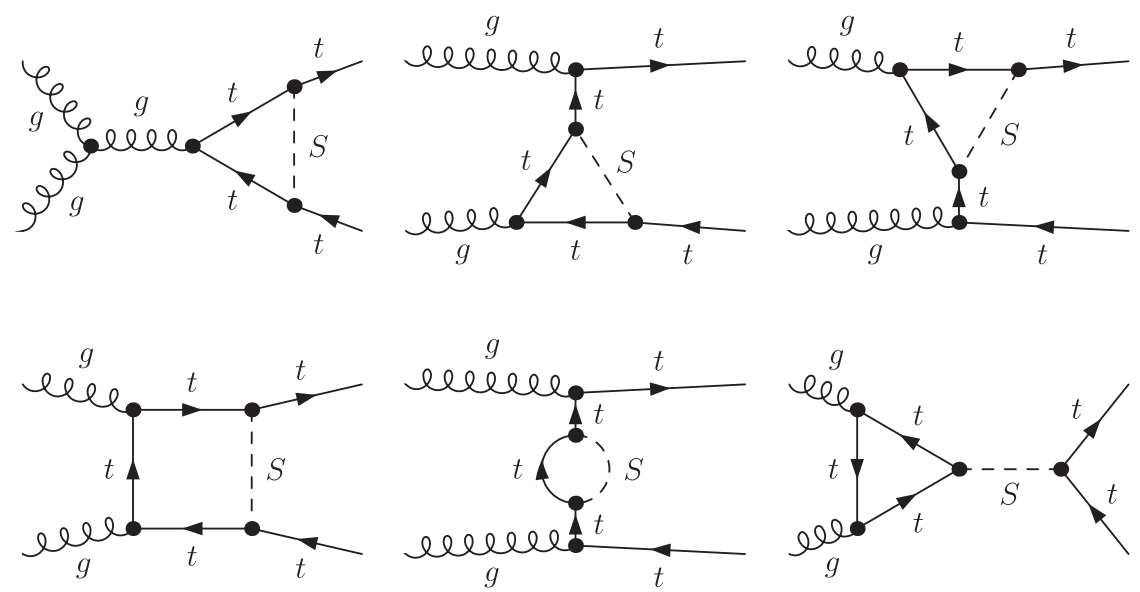

FIG. 1. Representative one-loop Feynman diagram contributions to $g g \rightarrow t \bar{t}$ arising in the simplified model of Eq. (1).

the gluon-induced process $g g \rightarrow t \bar{t}$ are shown in Fig. 1, where we do not consider SM electroweak contributions [60] (see also [61,62]). In the SM, for heavy Higgs bosons, it is known that the Higgs signal (with a large QCD $K$ factor $[63,64])$ has sizable interference effects with the QCD continuum in $g g \rightarrow t \bar{t}$ [65-67]. This influences exclusion constraints, but is also a viable source for new physics beyond the SM [20,68-77]. The predominant focus of previous work was therefore devoted to isolating the resonance shape and cross section, which is not our focus here. Note, however, that loop effects and their relation to (Higgs) effective field theory were first discussed in [78-81].

For our analysis, we implement the leading order, virtual, and counterterm (Fig. 2) contributions for $q \bar{q}, g g \rightarrow t \bar{t}$ production at $\mathcal{O}\left(c_{S}^{2}\right)$ in a modified version of VBFNLO [82-85] which links FORMCALC/LOOPTOOLS [86,87]. Various analytical comparisons against alternative calculations as well as numerical cross-checks of leading order amplitudes have been performed using MADGRAPH [88]. We use the on-shell renormalization scheme and have verified UV finiteness both analytically and numerically for the $g g$ and $q \bar{q}$ channels independently. We use real masses throughout this work, but note that the discrimination of signal and background can have shortfalls when the scalar width becomes comparable to the resonance mass [89-93], which is indicative of a loss of perturbative control [94].

We now turn to the effective theory description of the model of Eq. (1) at low energies or, equivalently, when the scalar mass $m_{S}$ is taken to be large. Integrating out the heavy scalar generates two dimension six operators that enter the processes considered in this paper. The first of these is a modified gluon- $t \bar{t}$ interaction, described by the effective operator

$$
\mathcal{O}_{t G}=v \bar{t}_{L} T^{a} \sigma^{\mu \nu} t_{R} G_{\mu \nu}^{a}
$$

(and its Hermitian conjugate). Here $t_{L}$ and $t_{R}$ denote left-handed and right-handed top quarks, $T^{a}$ are the $\mathrm{SU}(3)$ generators in the fundamental representation, $\sigma^{\mu \nu}=$ $i\left[\gamma^{\mu}, \gamma^{\nu}\right] / 2$, and $G_{\mu \nu}^{a}$ is the QCD gauge field strength tensor. Note that we have scaled the operator by an additional factor of the vacuum expectation value $v$ of the SM Higgs boson. The second operator is a four-fermion operator involving four top quarks and is given by expanding the
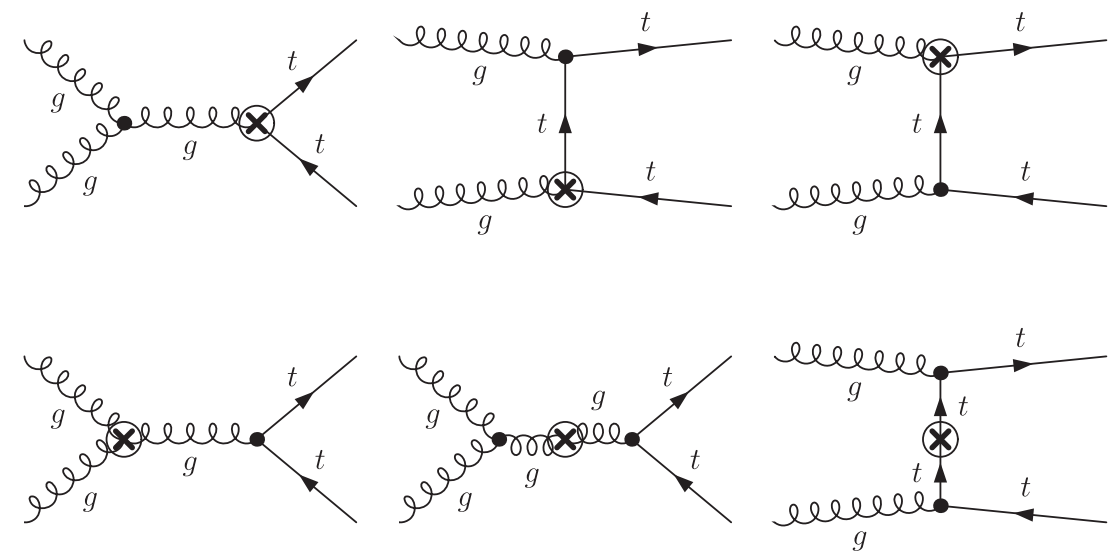

FIG. 2. Representative counterterm contributions to $g g \rightarrow t \bar{t}$. 


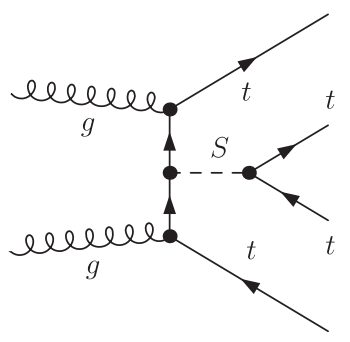

(a)

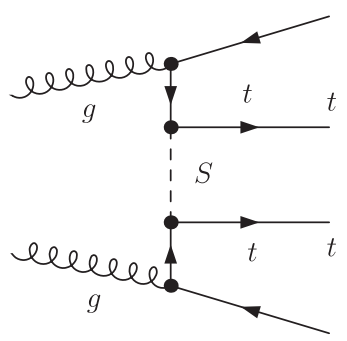

(b)

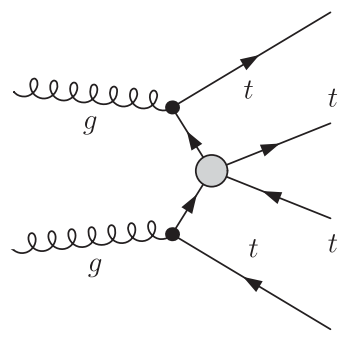

(c)

FIG. 3. (a), (b) Tree-level graph in the theory of Eq. (1) contributing to four top production; (c) tree-level contribution in the EFT description upon integrating out the heavy scalar, where the grey blob represents the operator of Eq. (4).

scalar propagator for large $m_{S}$ in relation to its four momentum $q^{2}$,

$$
(\bar{t} t) \frac{c_{S}^{2}}{q^{2}-m_{S}^{2}}(\bar{t} t) \stackrel{q^{2} \ll m_{S}^{2}}{\longrightarrow}-\frac{c_{S}^{2}}{m_{S}^{2}}(\bar{t} t)^{2}=\frac{c_{t t}}{\Lambda^{2}} \mathcal{O}_{t t}
$$

see e.g., Fig. 3. Note that this operator is not part of the Warsaw (SM EFT) basis [7], but it is more convenient for our purposes. For instance in four top production, the operator of Eq. (4) enters at tree level in the EFT, as illustrated in Fig. 3. The contribution from the operator $\mathcal{O}_{t G}$ is suppressed with respect to the contribution from $\mathcal{O}_{t t}$ because it is loop-induced and four top contributions with one $\mathcal{O}_{t G}$ insertion are of higher order in $\alpha_{s}$ than four top contributions with one insertion of $\mathcal{O}_{t t}$ (see Appendix). The situation is different in top pair production. Since $\mathcal{O}_{t t}$ enters only through loops (see e.g., Fig. 4) there is no relative suppression with respect to $\mathcal{O}_{t G}$ which is also loop-induced. Furthermore, tree-level diagrams with one $\mathcal{O}_{t G}$ insertion (whose topology is the same as the upper three diagrams in Fig. 2) and one-loop diagrams with one $\mathcal{O}_{t t}$ insertion (as in Fig. 4) contribute to the same perturbative order in $\alpha_{s}$. Hence, in top quark pair production both operators are relevant and have to be included in a consistent EFT calculation.
In particular, including the four top contribution requires the calculation of the loop diagrams of Fig. 4. These contributions require additional UV counterterms which are not present in the full theory but which renormalize the EFT operators. For illustrative purposes, we show the counterterm graphs in the gluon channel in Fig. 2. Renormalization of the UV divergences related to EFT operators is performed in the $\overline{\mathrm{MS}}$ scheme, and we have checked UV finiteness of all of our expressions for the final amplitude. The sum of tree-level contributions from $\mathcal{O}_{t G}$ and the one-loop contributions from $\mathcal{O}_{t t}$ yields the results for observables of top quark pair production at NLO EFT in terms of the Wilson coefficients $c_{t G}$ and $c_{t t}$.

Furthermore, our use of a specific model of new physics at high energy means that we can fix the values of the Wilson coefficients by matching the full theory and NLO EFT calculations at a suitable matching scale $\mu_{M}$ taking the operator mixing between $\mathcal{O}_{t G}$ and $\mathcal{O}_{t t}$ into account. We choose $\mu_{M}=m_{S} / 2$ in the following unless otherwise specified. We extract $c_{t G}$ as the finite remainder after subtracting the $\overline{\mathrm{MS}}$-renormalized four fermion oneloop insertion from the EFT operator that is induced by the propagating $S$. Note that it does not require UV renormalization as opposed to the four fermion insertion.
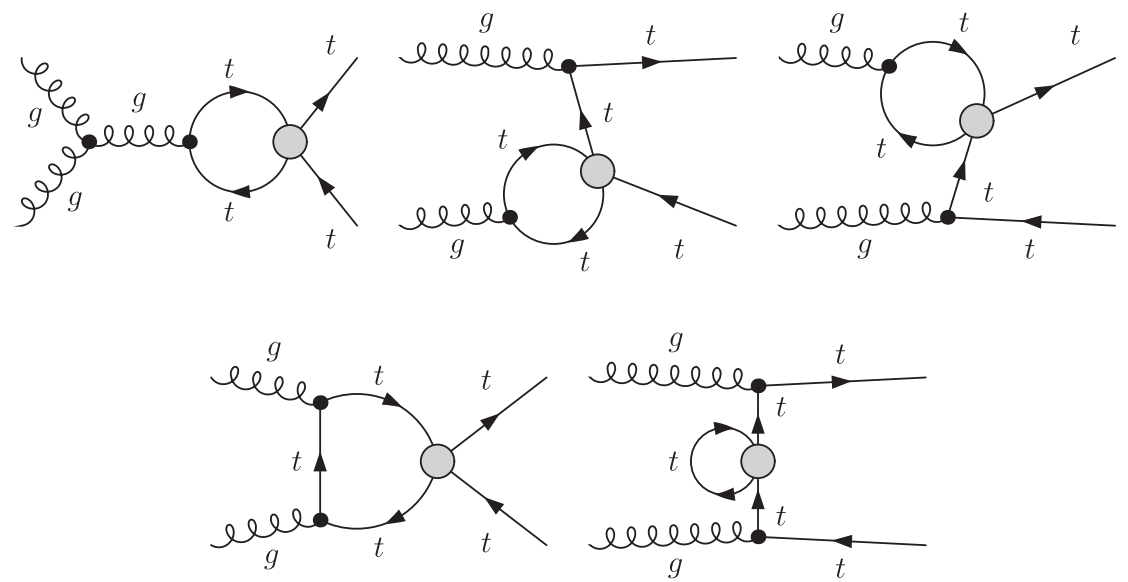

FIG. 4. Representative one-loop Feynman diagram contributions to $g g \rightarrow t \bar{t}$ arising in the effective theory formulation of Eq. (1); the shaded region represents a four top insertion. 

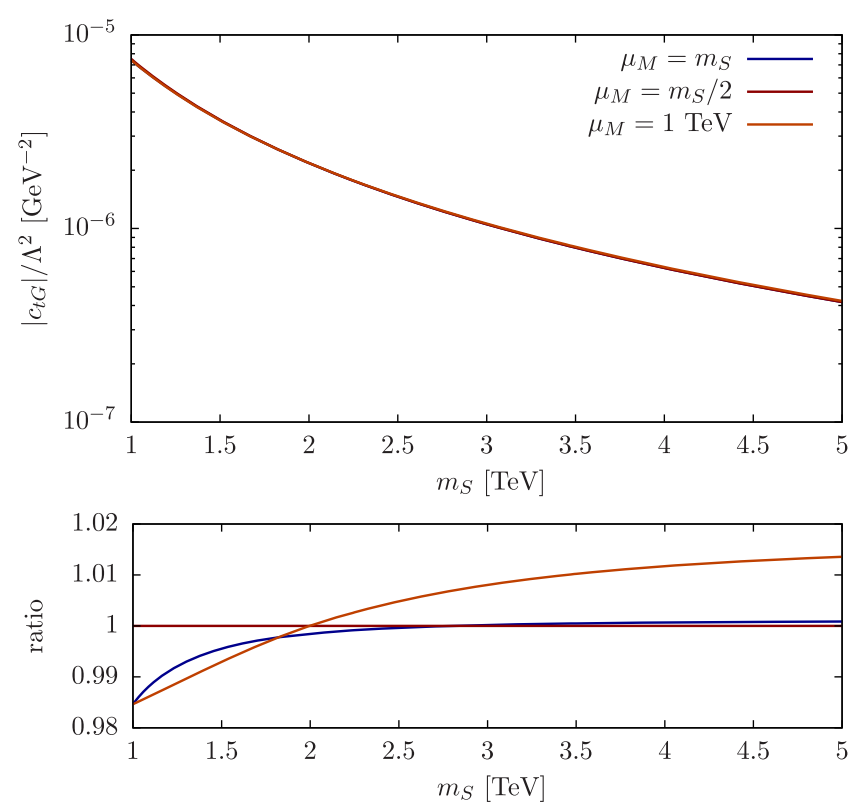

FIG. 5. Matched value of $c_{t G}$ for different matching scale choices and scalar masses $\left(c_{S}=1\right)$ as detailed in the text.

The dependence of $c_{t G}$ on the matching scale is shown in Fig. 5. As the matching scale is related to a renormalization scale choice (Appendix), the cross section has a logarithmic dependence on the $\mu_{M}$.

As for the full simplified model calculation described above, we have implemented our matched NLO calculation in a modified version of VBFNLO [82-85].
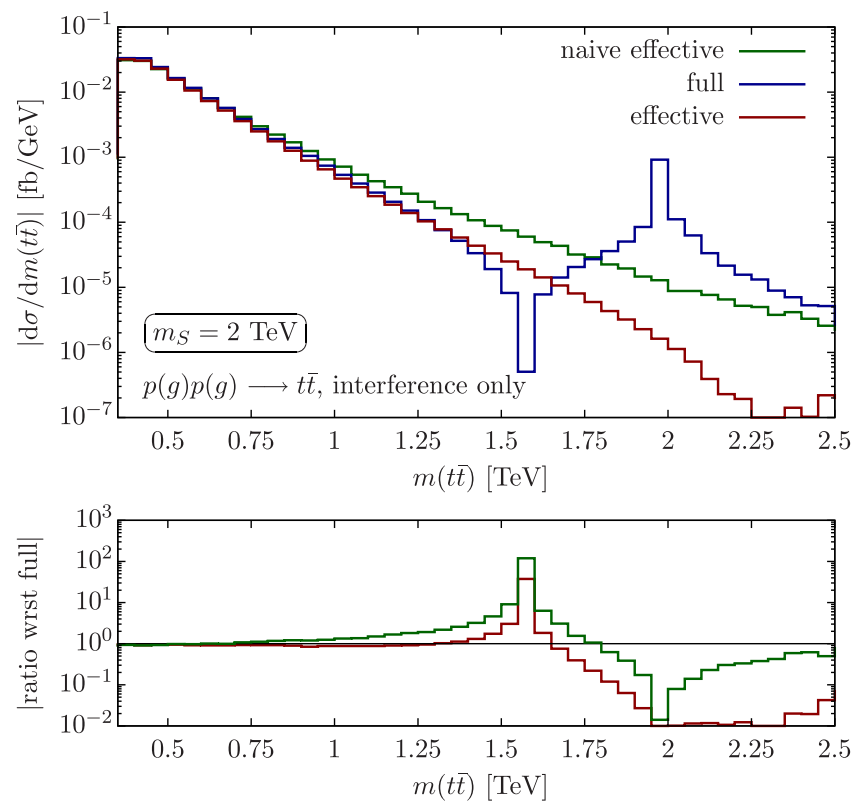

\section{VALIDITY OF EFT AT (NEXT-TO-)LEADING ORDER}

In the previous section, we outlined a particular simplified model for new physics in the top quark sector and explained how this can be matched to an EFT description at low energies. In this section, we analyze the range of validity of the latter, as the mass of the scalar particle is lowered toward LHC energies. We will illustrate our results using the invariant mass distribution of the final state tops in top pair production, although similar results would be obtained for other kinematic distributions.

In Fig. 6, we show the contribution to the invariant mass $m_{t \bar{t}}$ stemming from the interference between the new physics process and the SM contribution,

$$
\mathrm{d} \sigma(t \bar{t}) \sim 2 \operatorname{Re}\left(\mathcal{M}_{\bar{t} \bar{t}}^{\mathrm{SM}} \mathcal{M}_{t \bar{t}}^{* \mathrm{virt} / \mathrm{d} 6}\right),
$$

where virt/d6 represents the propagating- $S$ contributions or their dimension six EFT counterparts, for a scalar mass of $m_{S}=2 \mathrm{TeV}$. Three different curves are shown. The blue curve shows the result obtained from the full theory of Eq. (1), with all dynamics correctly included. The red curve shows the results of our NLO-matched EFT calculation. We see that the EFT and the full computation agree well, as long as we are away from the turn-on of the scalar BreitWigner distribution. The green curve in Fig. 6 shows the results of a bottom-up approach to EFT where we assume no knowledge about the full theory. Specifically, we perform a LO EFT calculation of $t \bar{t}$ production taking only tree-level diagrams with one insertion of $\mathcal{O}_{t G}$ into
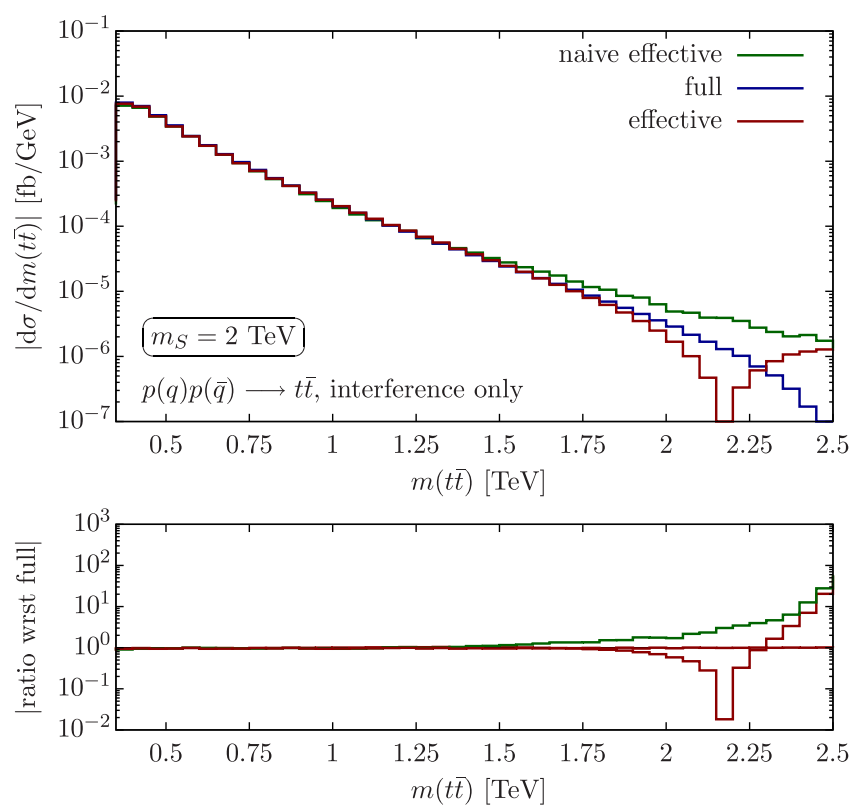

FIG. 6. BSM interference contribution as a function of the invariant $t \bar{t}$ mass for gluon fusion (left) and $q \bar{q}$ annihilation, neglecting the $Z$ contribution. As the interference changes sign we choose to plot the absolute value of the interference for clarity. We choose $m_{S}=2 \mathrm{TeV}$ and $c_{S}=0.1$. 

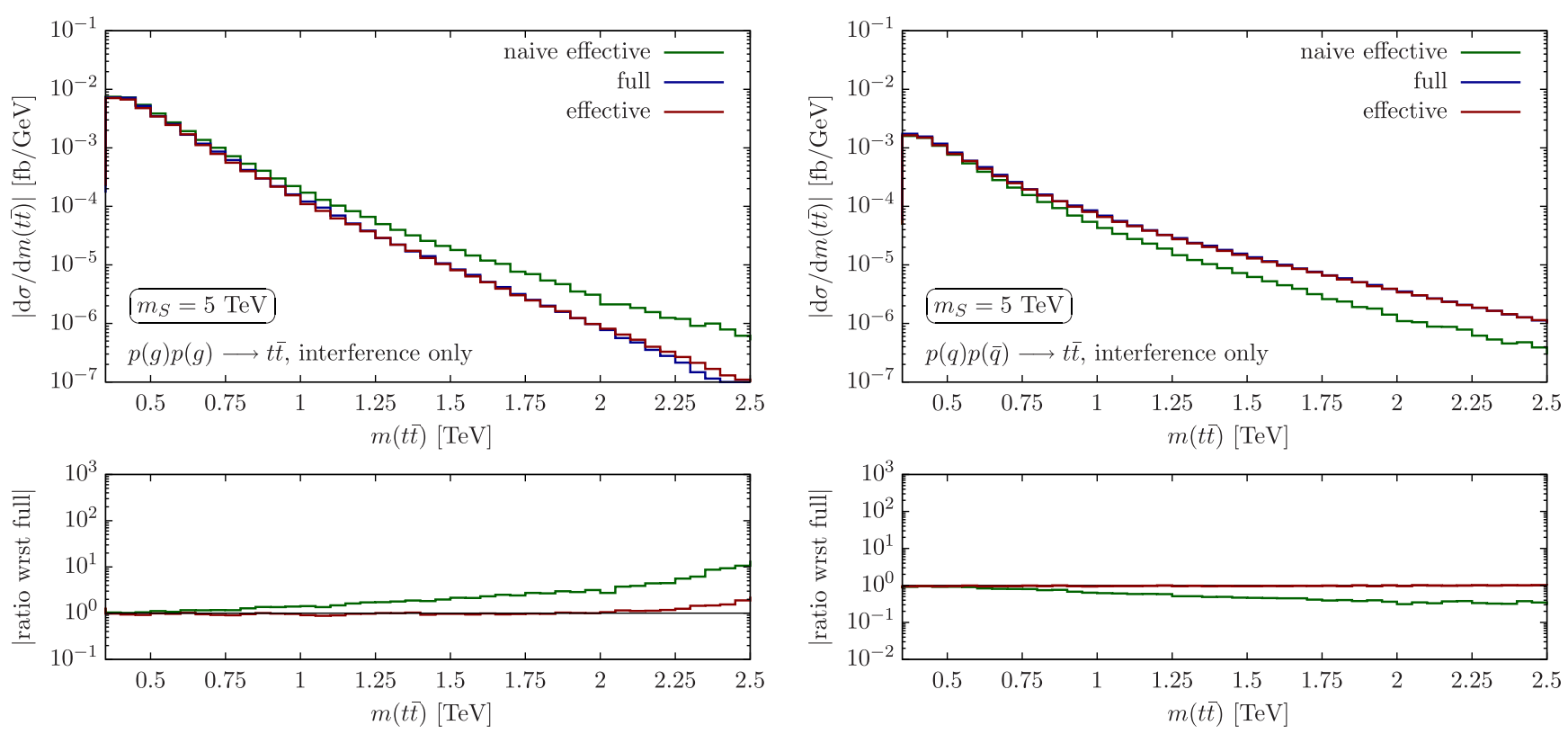

FIG. 7. As Fig. 6 but choosing $m_{S}=5 \mathrm{TeV}$ and again $c_{S}=0.1$ to demonstrate the broad range of agreement for heavy scalar masses probing the LHC kinematical coverage.

account. We treat the Wilson coefficient $c_{t G}$ as a free parameter in the EFT and fit $c_{t G}$ to Monte Carlo data that were generated using the full theory. This approach simulates an EFT fit where the EFT prediction is calculated at LO and applied to data which contain the signatures of the simplified model of Eq. (1). This naïve approach based on fitting $c_{t G}$ alone never reproduces the correct shape. This becomes even more transparent when we push the scalar mass to larger values, e.g., $m_{S}=5 \mathrm{TeV}$ in Fig. 7. The full theory and the NLO EFT calculation agree very well, with the turn-on of the scalar exchange only leading to mild corrections for large values of $m(t \bar{t})$ in the (dominant) gluon fusion component. Again as expected, the LO EFT approach now deviates significantly. In particular, fixing the coefficient of $c_{t G}$ at low energies where the $m_{t \bar{t}}$ distribution is measured more precisely leads to a drastic mismodeling of the shape of the invariant mass distribution, with a significant overestimate of the high mass tail. As we will see in the following section, this can lead to an overly optimistic constraint on possible new physics effects, for the model that we consider here.

In Fig. 8, we indicate the validity range when comparing full theory and NLO EFT computation (for a general discussion see [95]). The parameter $m^{\max }(t \bar{t})$ denotes the energy scale at which the NLO EFT and full computations deviate beyond the indicated percentages for $c_{S}=1$. In this comparison we also include the squared resonance contribution. Note that for this coupling choice, the width remains at $\simeq 0.1 m_{S}$ leading to the turn-on of the BreitWigner distribution becoming resolvable in the direct comparison. This turn-on cannot be resolved when background uncertainties are included (see below).
Our results in this section confirm the possibility of obtaining an accurate EFT description of the simplified model of Eq. (1), which is generic enough to apply in multiple contexts, including singlet Higgs mixing scenarios and multi-Higgs doublet extensions. A key issue facing contemporary global EFT fits is whether to pursue the effort of carrying out a full NLO calculation for all processes and observables considered. The latter requires a considerable effort (see e.g., [37,96] for recent examples), although the intermediate possibility also exists of including renormalization group mixing effects between dimension six operators, but neglecting additional contributions

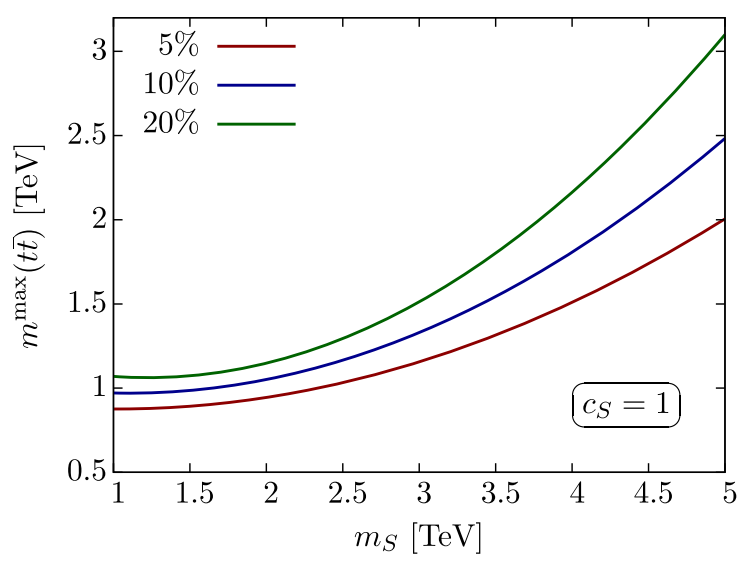

FIG. 8. Validity within percentage of the EFT computation when compared to the full model including squared Higgs resonance contribution. $m^{\max }(t \bar{t})$ indicates the invariant (anti) top mass, at which the relative difference becomes larger than the indicated percentage. 
that are nonlogarithmic in the matching scale. The importance of NLO effects in the present case is ultimately due to the fact that of the two operators that are sourced in the low energy description, one is tree-induced but the other is loop-induced. Our example thus clearly shows the need to bear such considerations in mind when trying to match EFT constraints to specific new physics models.

\section{LHC COMPARISONS, RESULTS, AND EXTRAPOLATIONS}

Both top pair and four top production are being actively measured at the LHC and will play a crucial role in searching for new physics in the top quark sector in the coming years. To this end, it is instructive to examine the sensitivity that the LHC is likely to achieve following its high luminosity upgrade, with an expected $3 \mathrm{ab}^{-1}$ of data. We will do this here for two scenarios. First, we will constrain the full simplified model of Eq. (1) directly. Assuming a given uncertainty for the above-mentioned processes leads to exclusion contours in the $\left(m_{S}, c_{S}\right)$ parameter space, shown in Fig. 9, where anything above a given curve (i.e., for stronger couplings $c_{S}$ ) is excluded. Second, we will assume that an NLO EFT analysis has been applied, leading to constraints on the coefficients of the new physics operators $\mathcal{O}_{t G}$ and $\mathcal{O}_{t t}$. By matching with the full theory as described previously, constraints on the

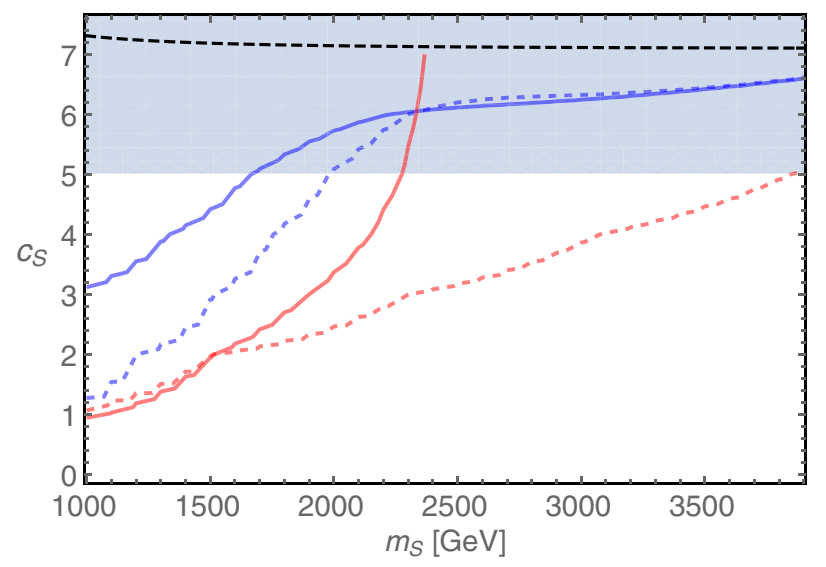

FIG. 9. The 95\% confidence level exclusion contours for the simplified model of Eq. (1) as a function of its mass $m_{S}$ and top coupling $c_{S}$. The blue solid contour shows the full result (i.e., propagating $S$ at NLO) while the blue dashed line corresponds to the EFT calculation. For $p p \rightarrow t \bar{t}$ we assume a flat uncertainty of $3 \%$. The solid red line represents a $p p \rightarrow t \bar{t} t \bar{t}$ analysis of the simplified scenario using the extrapolation of Ref. [56] while the red dashed line represents the (LO) EFT four top results. The shaded band shows the region where perturbative unitarity is lost, $c_{S} \gtrsim \sqrt{8 \pi}$ which we obtain from an explicit partial wave projection calculation of $t \bar{t} \rightarrow t \bar{t}$ in the full model, i.e., with propagating $S$. Note that this is precisely the region where $\Gamma(S \rightarrow$ $t \bar{t}) \simeq m_{S}$ according to Eq. (2). Finally, the black dashed line is the unitarity constraint on the effective four top interaction, below which unitarity is preserved (for details see text). operator coefficients can also be converted to curves in the $\left(m_{S}, c_{S}\right)$ plane.

The top pair production cross section is currently known at NNLO precision [97,98] (see also [99]). Given the large cross section, the theoretical uncertainty will be the limiting factor of physics in the top sector (see also [24]). In Fig. 9, we show the sensitivity of the LHC under the assumption that the unfolded $m_{t \bar{t}}$ distribution can be described at an optimistic 3\% level using a binned $\chi^{2}$ test as detailed in Ref. [15]. For this particular error choice the EFT and full theory agreement happens to be slightly above the perturbative unitarity limit of $c_{S}^{2} \simeq 8 \pi$ that can be derived from $t \bar{t} \rightarrow t \bar{t}$ scattering in the full model (i.e., with propagating $S$ ). A larger error budget quickly pushes the constraints deeply into the nonperturbative regime. On the other hand, sensitivity to $c_{S} \simeq 1$ requires per mille level uncertainties. These are beyond the current state-of-the-art. As can be seen, for large scalar masses where the EFT reproduces the full model expectations both approaches are compatible. At lower masses, tighter constraints are obtained in the EFT than in the full theory. This is due to the systematic tendency (visible in Figs. 6 and 7) of the EFT to overestimate the full theory due to the absence of absorptive parts in the region where the scalar contribution gets resolved. Thus, applying EFT alone would result in overly optimistic reported constraints on new physics that would not be strictly valid. Note that in this comparison we include the squared $s$-channel scalar contribution with an approximate $K$ factor $\simeq 2.5[63,64]$ as this significantly impacts the exclusion for the dynamic $S$. Notwithstanding the accuracy at which the EFT manages to approximate the full computation, we see that hadron collider systematics do seriously curtail precision physics in the top sector when contrasted with certain classes of top-philic BSM models. The simplified model highlights this through Fig. 9. Gaining sensitivity in such an instance crucially rests on more precise SM predictions that allow constraints to be pushed into the perturbative limit of the model.

One might argue that discovering a contrived top-philic new physics scenario is difficult to achieve in the first place. However, for the scenario that we have studied there is the possibility to investigate four top final states similar to existing analyses [26,50,51]. The experiments have also performed extrapolations to the HL-LHC, e.g., [55-57]. As the cross sections for this process are relatively small, $\mathcal{O}(10 \mathrm{fb})[100,101]$, statistical and experimental uncertainties will be important. There is reason to believe that the latter can be brought under sufficient control and e.g., ATLAS have shown that a sensitivity of $11 \%$ around the SM expectation can be achieved [56] which is smaller than the current theoretical precision. It is not unreasonable to expect that theoretical predictions can be improved, and we assume a $18 \%$ accuracy in the extraction of the unfolded $t \bar{t} t \bar{t}$ cross section, which is slightly worse than the ATLAS extrapolation and the lowest bound provided by CMS [55]. 
We simulate four top events using MadEvent [88] keeping track of destructive interference effects that arise between the QCD and new scalar contributions. In the four top case, these are much smaller than for $g g \rightarrow t \bar{t}$, and we find a typical mild correction of $\mathcal{O}(-10 \%)$. Constraints on the parameter space from applying the full simplified model, and the EFT approach, are shown in Fig. 9. Given that there is a tree-level-induced dimension six operator in this process (i.e., the four-fermion operator), we restrict the present discussion to LO only. For low scalar masses the constraints are comparable. However, for larger masses applying the full model directly leads to very weak constraints. This behavior is dominated by the assumed uncertainties, coupled with the fact that at higher masses in the full theory, the decay width of the scalar [from Eq. (2)] becomes large. This decreases the scalar contribution to four top final states to a large extent, leading to a loss of sensitivity for the simplified model in four top final states under our assumptions at around $m_{S} \simeq 2.2 \mathrm{TeV}$. The BreitWigner cross-section distribution of the $t \bar{t}$ system for large enough $c_{S}$ behaves as $\sim \tilde{\Gamma}^{-2}$ [see Eq. (2)], i.e., flattens out as a function of $c_{S}$ such that the cross-section constraint for large enough $c_{S}$ is determined by the mass $m_{S}$. This means that the sensitivity translated to our simplified model calculation is no longer under perturbative control. ${ }^{2}$ This effect is mainly driven by the dynamics in the full theory of the chosen model and is not a problem of setting constraints using EFT approaches. Hence, applying a cut on the typical energy scale of the process as proposed for example in [95] would not resolve this problem. While the high mass region is plagued by large width effects in the full theory, the lower masses $(<2 \mathrm{TeV})$ shown in Fig. 9 are accessible at the LHC. Hence, matching to the full scenario is not possible in a perturbatively meaningful way as mentioned above and illustrated by the discrepancy between the blue solid and dashed lines in Fig. 9 in the low mass region. The relatively good agreement between the red solid and dashed lines in that mass region is accidental.

However, without considering a specific UV model we can check the self-consistency of the EFT with respect to perturbative unitarity. Such constraints arise from four top scattering in the EFT, i.e., through the four top contact interaction in Eq. (4), and are given by

$$
c_{S}^{2} \lesssim 16 \pi \frac{m_{S}^{2}}{E_{\max }^{2}-2 m_{t}^{2}},
$$

where, for illustrative purposes, we have matched the constraint on $c_{t t}$ to $c_{S}$ using Eq. (4). $E_{\max }$ denotes the

\footnotetext{
${ }^{2}$ The width being related to the resummation of the imaginary part of the top 2 point function signifies the relevance of higher order corrections in this model for the four top final states as well. These effects would be interesting to study but are beyond the scope of this work.
}

energy where unitarity is broken in the leading order approximation. This happens at the latest at $E_{\max } \simeq m_{S}$ when considering our scenario, which is depicted by the black dashed line in Fig. 9. Comparing this bound with the red dashed curve shows that the EFT constraints on $c_{S}$ (or rather $c_{t t}$ ) from four top final states remain within the perturbative unitarity bounds of the EFT. Hence, analyses of the four top final states do give rise to constraints which are perturbatively meaningful in different scenarios where matching is possible (e.g., [102-104]).

\section{SUMMARY AND CONCLUSIONS}

Effective field theory approaches are becoming a new standard for the dissemination of LHC physics results. In contrast to flavor physics where EFT methods have been successfully employed over decades (see e.g., [105]), the nonobvious scale separation of hadron collider measurements that probe a broad partonic center of mass energy range makes their implementation less straightforward. In particular, operator mixing effects that are sensitive to whether dimension six operators are tree- or loop-induced in particular UV scenarios will shape the phenomenology at intermediate scales and has to be reflected consistently in any limit setting procedure. In this work we have examined a particular scalar simplified model with top-philic couplings that approximates a broad range of UV scenarios, with the particular aim to gauge the sensitivity reach of top quark final states at the LHC. Top pair production processes with large cross sections are prime candidates to look for new physics effects with statistical control. We demonstrate that the NLO matching of the EFT and full model allows a broad range of agreement of the two approaches, up to $\sim 3 \mathrm{TeV}$ (in e.g., the top pair invariant mass distribution) for order one coupling choices of the simplified model. This agreement can be pushed higher when couplings are such as to reduce the Breit-Wigner-induced departure for invariant masses below the resonance threshold. For our simplified scenario we find that systematic limitations of top pair measurements dilute the sensitivity to new physics effects in particular in the region where the EFT approach (which is nontrivial in this context) provides an excellent approximation to the UV theory. In this sense, repeating the Higgs discovery success story where precision effects allowed to constrain the Higgs' presence marginally outside LEP's kinematic coverage seems unlikely when $p p \rightarrow t \bar{t}$ is considered in isolation (i.e., no other competing BSM effects are present). Pivotal to changing this situation is the continued precision calculation efforts for SM processes, and $t \bar{t}$ production in particular in our context. In the concrete case of top-philic interactions as expressed by the scalar model, subsidiary measurements such as four top final states can provide additional sensitivity. While these processes are considerably rarer than top pair production at the LHC, they have direct sensitivity to four top contact interactions which are clear signs of top-philic 
interactions below their characteristic scale. Including four top final states in leading order fits is therefore crucial to achieve sensitivity to the scenario discussed in this work, as an example for new physics that predominantly talks to the top sector.

\section{ACKNOWLEDGMENTS}

This work was supported by the Munich Institute for Astro- and Particle Physics (MIAPP) of the DFG Excellence Cluster Origins (www.origins-cluster.de). C. E. and P. G. are supported by the UK Science and Technology Facilities Council (STFC), under Grant No. ST/P000746/1. C. D. W. is supported by the STFC, under Grant No. ST/P000754/1 and by the European Union Horizon 2020 research and innovation programme under the Marie Skłodowska-Curie Grant Agreement No. 764850 "SAGEX."

\section{APPENDIX: NOTES ON RENORMALIZATION AND MATCHING}

The UV divergent corrections of top pair production in the simplified model are given by the vertex and propagator corrections depicted in Fig. 1. The on-shell renormalization of UV divergencies is determined only by top quark mass and wave function counterterms (these can be found in Ref. [106]). The cancellation of UV singularities along these lines is expected by the gaugesinglet character of $S$ and the product-group gauge theory form of the SM. Hence, there is no renormalization of the gauge couplings.

The qualitative changes in the renormalization procedure when comparing full and effective theory computation is highlighted by considering the top quark two-point function. Approaching the limit $m_{S} \rightarrow \infty$ before carrying out the loop integration results in a schematic identification

$$
\stackrel{B_{0}\left(q^{2}, m_{t}, m_{S}\right) \sim \longrightarrow}{\longrightarrow} \longrightarrow \frac{A_{0}\left(m_{t}\right)}{m_{S}^{2}}
$$

where $A_{0}$ and $B_{0}$ are the Passarino-Veltman one-point and two-point scalar functions $[106,107]$. Since the $A_{0}$ function does not depend on the momentum of the two-point function there is no top quark wave function renormalization involved in the EFT calculation. Instead the renormalization of the EFT calculation is performed in the top quark mass and the Wilson coefficient $c_{t G}$. The EFT renormalization of the top mass due to the four fermion insertion is given by

$$
\delta m_{t}^{\mathrm{EFT}}=\frac{c_{t t}}{16 \pi^{2} \Lambda^{2}} m_{t} A_{0}\left(m_{t}\right)
$$

The one-loop EFT contributions (see Fig. 4) give rise to UV singularities. After top mass renormalization we are left with the following UV divergence in the NLO EFT amplitude

$$
\left.\mathcal{M}(g g \rightarrow t \bar{t})\right|_{\mathrm{NLO}, \mathrm{div}} ^{\mathrm{EFT}, m_{t} \text {-ren }}=-\frac{c_{t t} g_{s} y_{t}}{32 \pi^{2} \Lambda^{2}} \Delta^{\mathrm{UV}}\left\langle\mathcal{O}_{t G}\right\rangle,
$$

where $\Delta^{\mathrm{UV}}=\epsilon^{-1}-\gamma_{E}+\log 4 \pi$ in dimensional regularization with $D=4-2 \epsilon$ dimensions and $y_{t}$ denotes the top Yukawa coupling [we have traded $m_{t}$ against the vacuum expectation value that appears in the normalization of Eq. (3)]. The amplitude $\left\langle\mathcal{O}_{t G}\right\rangle$ denotes all $\mathcal{O}_{t G}$ operator insertions that contribute to $g g \rightarrow t \bar{t}$ at tree level including those with contact interactions $g g t \bar{t}$. This shows that the one-loop insertion of the four-fermion operator $\mathcal{O}_{t t}$ induces a renormalization of the $\mathcal{O}_{t G}$ operator since the LO EFT amplitude is given by

$$
\left.\mathcal{M}(g g \rightarrow t \bar{t})\right|_{\mathrm{LO}} ^{\mathrm{EFT}}=\left\langle\mathcal{O}_{\mathrm{SM}}\right\rangle+\frac{c_{t G}}{\Lambda^{2}}\left\langle\mathcal{O}_{t G}\right\rangle
$$

where $\left\langle\mathcal{O}_{\mathrm{SM}}\right\rangle$ represents the SM amplitude, which is independent from $\left\langle\mathcal{O}_{t G}\right\rangle$ as a result of [7]. The divergence in Eq. (A3) can be removed by including a $c_{t G}$ counterterm

$$
\frac{\delta c_{t G}}{\Lambda^{2}}=\frac{c_{t t} g_{s} y_{t}}{32 \pi^{2} \Lambda^{2}}\left(\Delta^{\mathrm{UV}}+\mathcal{F}\left(\mu^{2}\right)\right),
$$

where $\mathcal{F}$ denotes renormalization-scheme dependent finite terms that will be fixed when we match the one-loop EFT amplitude with the on-shell renormalized one-loop result for propagating $S$ at a matching scale $\mu_{M}$. The matching relation (which also addresses the quark-induced channels) is given by
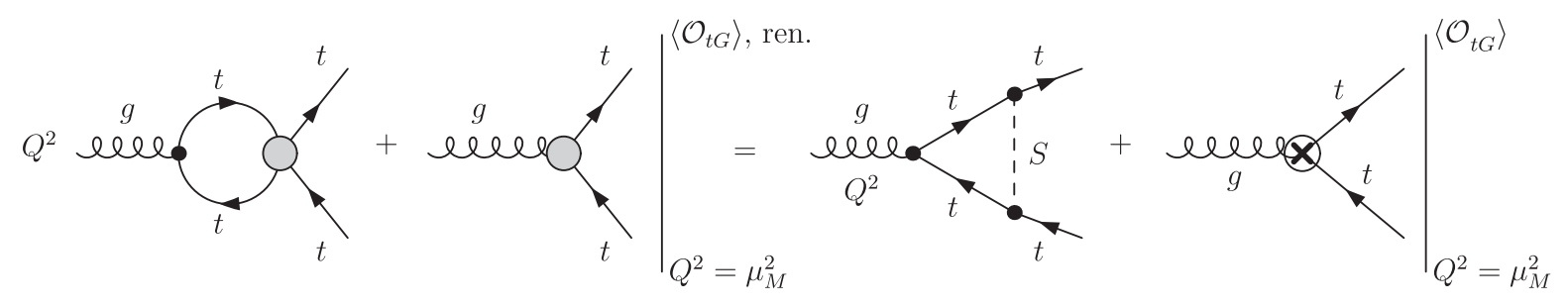
Concretely this means that we first extract the Lorentz structure related to the operator insertion of $\mathcal{O}_{t g}$ of the renormalized EFT as well as the full calculation. We then identify the coefficients of the $\mathcal{O}_{t g}$ amplitudes (Lorentz structures) at a matching scale $\mu_{M}^{2}$, which fixes the finite terms $\mathcal{F}\left(\mu_{M}^{2}\right)$ that correspond to a tree-level insertion of $\mathcal{O}_{t G}$ after matching

$$
\begin{aligned}
\mathcal{F}\left(\mu_{M}^{2}\right)= & -\frac{m_{S}^{2}}{\mu_{M}^{2}-4 m_{t}^{2}}+\frac{m_{S}^{2}}{\mu_{M}^{2} m_{t}^{2}-4 m_{t}^{4}} \tilde{A}_{0}\left(m_{S}^{2}\right)-\frac{m_{S}^{2}}{m_{t}^{2}\left(\mu_{M}^{2}-4 m_{t}^{2}\right)} \tilde{A}_{0}\left(m_{t}^{2}\right) \\
& +\frac{m_{S}^{2}\left(4 m_{t}^{2}\left(\mu_{M}^{2}-4 m_{t}^{2}\right)-m_{S}^{2}\left(\mu_{M}^{2}-10 m_{t}^{2}\right)\right)}{m_{t}^{2}\left(\mu_{M}^{2}-4 m_{t}^{2}\right)^{2}} \tilde{B}_{0}\left(m_{t}^{2}, m_{S}^{2}, m_{t}^{2}\right)+\left(1-\frac{3 m_{S}^{2}\left(2 m_{S}^{2}+\mu_{M}^{2}-4 m_{t}^{2}\right)}{\left(\mu_{M}^{2}-4 m_{t}^{2}\right)^{2}}\right) \tilde{B}_{0}\left(\mu_{M}^{2}, m_{t}^{2}, m_{t}^{2}\right) \\
& -\frac{6 m_{S}^{4}\left(m_{S}^{2}+\mu_{M}^{2}-4 m_{t}^{2}\right)}{\left(\mu_{M}^{2}-4 m_{t}^{2}\right)^{2}} \tilde{C}_{0}\left(m_{t}^{2}, \mu_{M}^{2}, m_{t}^{2}, m_{S}^{2}, m_{t}^{2}, m_{t}^{2}\right)
\end{aligned}
$$

where $\tilde{A}_{0}, \tilde{B}_{0}$, and $\tilde{C}_{0}$ are the $\epsilon \rightarrow 0$ finite remainders of the Passarino-Veltman one-, two-, and three-point scalar integrals, respectively, in the convention of $[86,87]$. This gives rise to a matched value

$$
\frac{c_{t G}\left(\mu_{M}^{2}\right)}{\Lambda^{2}}=-\frac{c_{S}^{2} g_{s} y_{t}}{32 \pi^{2} m_{S}^{2}} \mathcal{F}\left(\mu_{M}^{2}\right),
$$

which reflects the $g_{s}$-loop-induced nature of $c_{t G}$ in the considered simplified model. The inclusion of appropriately defined finite terms in the comparison of Sec. II is crucial to obtain agreement between full and EFT-based computation. This balances the $c_{t G}$-related momentum transfer behavior of $\left\langle\mathcal{O}_{t G}\right\rangle$ against the virtual contributions of $\mathcal{O}_{t t}$. In a naïve or bottom-up approach based on $\left\langle\mathcal{O}_{t G}\right\rangle$ alone without matching this balance is lost which leads to overestimates in the tails of distributions long before $\sqrt{s} \simeq m_{S}$.

Note that only the sum of loop-inserted $c_{t t}$ and tree-level $c_{t G}$ is defined as a consequence of Eq. (A6), and we can always move finite terms between the EFT coefficients. The scheme that we adopt is fixing $c_{t t}$ through leading order $t \bar{t} \rightarrow t \bar{t}$ scattering (accessible in four top final states), which leaves $c_{t G}$ determined as a function of $\mu_{M}$.
[1] K. Kröninger, A. B. Meyer, and P. Uwer, in The Large Hadron Collider: Harvest of Run 1, edited by T. SchörnerSadenius (Springer, Cham, 2015), pp. 259-300, https:// link.springer.com/chapter/10.1007\%2F978-3-319-150017 7.

[2] S. Weinberg, Physica (Amsterdam) 96A, 327 (1979).

[3] W. Buchmuller and D. Wyler, Nucl. Phys. B268, 621 (1986).

[4] C. J. C. Burges and H. J. Schnitzer, Nucl. Phys. B228, 464 (1983).

[5] C. N. Leung, S. T. Love, and S. Rao, Z. Phys. C 31, 433 (1986).

[6] K. Hagiwara, R. D. Peccei, D. Zeppenfeld, and K. Hikasa, Nucl. Phys. B282, 253 (1987).

[7] B. Grzadkowski, M. Iskrzynski, M. Misiak, and J. Rosiek, J. High Energy Phys. 10 (2010) 085.

[8] A. Dedes, W. Materkowska, M. Paraskevas, J. Rosiek, and K. Suxho, J. High Energy Phys. 06 (2017) 143.

[9] I. Brivio and M. Trott, Phys. Rep. 793, 1 (2019).

[10] J. Alwall, P. Schuster, and N. Toro, Phys. Rev. D 79, 075020 (2009).

[11] D. Alves (LHC New Physics Working Group), J. Phys. G 39, 105005 (2012).

[12] J. Abdallah et al., Phys. Dark Universe 9-10, 8 (2015).
[13] A. Buckley, C. Englert, J. Ferrando, D. J. Miller, L. Moore, M. Russell, and C. D. White, Phys. Rev. D 92, 091501 (2015).

[14] M. P. Rosello and M. Vos, Eur. Phys. J. C 76, 200 (2016).

[15] A. Buckley, C. Englert, J. Ferrando, D. J. Miller, L. Moore, M. Russell, and C. D. White, J. High Energy Phys. 04 (2016) 015.

[16] C. Zhang, Phys. Rev. Lett. 116, 162002 (2016).

[17] O. B. Bylund, F. Maltoni, I. Tsinikos, E. Vryonidou, and C. Zhang, J. High Energy Phys. 05 (2016) 052.

[18] N. Castro, J. Erdmann, C. Grunwald, K. Kröninger, and N.-A. Rosien, Eur. Phys. J. C 76, 432 (2016).

[19] D. Barducci, M. Fabbrichesi, and A. Tonero, Phys. Rev. D 96, 075022 (2017).

[20] C. Englert and M. Russell, Eur. Phys. J. C 77, 535 (2017).

[21] M. Schulze and Y. Soreq, Eur. Phys. J. C 76, 466 (2016).

[22] J. L. Birman, F. Déliot, M. C. N. Fiolhais, A. Onofre, and C. M. Pease, Phys. Rev. D 93, 113021 (2016).

[23] V. Cirigliano, W. Dekens, J. de Vries, and E. Mereghetti, Phys. Rev. D 94, 034031 (2016).

[24] C. Englert, L. Moore, K. Nordström, and M. Russell, Phys. Lett. B 763, 9 (2016).

[25] F. Maltoni, E. Vryonidou, and C. Zhang, J. High Energy Phys. 10 (2016) 123. 
[26] C. Zhang, Chin. Phys. C 42, 023104 (2018).

[27] S. M. Etesami, S. Khatibi, and M. Mohammadi Najafabadi, Phys. Rev. D 97, 075023 (2018).

[28] D. Barducci et al., arXiv:1802.07237.

[29] M. Malekhosseini, M. Ghominejad, H. Khanpour, and M. M. Najafabadi, Phys. Rev. D 98, 095001 (2018).

[30] C. Degrande, F. Maltoni, K. Mimasu, E. Vryonidou, and C. Zhang, J. High Energy Phys. 10 (2018) 005.

[31] A. Jueid, Phys. Rev. D 98, 053006 (2018).

[32] J. D'Hondt, A. Mariotti, K. Mimasu, S. Moortgat, and C. Zhang, J. High Energy Phys. 11 (2018) 131.

[33] G. Durieux, M. Perelló, M. Vos, and C. Zhang, J. High Energy Phys. 10 (2018) 168.

[34] M. de Beurs, E. Laenen, M. Vreeswijk, and E. Vryonidou, Eur. Phys. J. C 78, 919 (2018).

[35] C. Englert, M. Russell, and C. D. White, Phys. Rev. D 99, 035019 (2019).

[36] M. Chala, J. Santiago, and M. Spannowsky, J. High Energy Phys. 04 (2019) 014.

[37] N. P. Hartland, F. Maltoni, E. R. Nocera, J. Rojo, E. Slade, E. Vryonidou, and C. Zhang, J. High Energy Phys. 04 (2019) 100.

[38] G. Durieux, A. Irles, V. Miralles, A. Peñuelas, R. Pöschl, M. Perelló, and M. Vos, J. High Energy Phys. 12 (2019) 098.

[39] R. P. Moutafis, Master's thesis, Orsay, LAL, 2019.

[40] W. Liu and H. Sun, Phys. Rev. D 100, 015011 (2019).

[41] R. Boughezal, C.-Y. Chen, F. Petriello, and D. Wiegand, Phys. Rev. D 100, 056023 (2019).

[42] T. Neumann and Z. E. Sullivan, J. High Energy Phys. 06 (2019) 022.

[43] F. del Aguila, Z. Kunszt, and J. Santiago, Eur. Phys. J. C 76, 244 (2016).

[44] Z. Zhang, J. High Energy Phys. 05 (2017) 152.

[45] S. A. R. Ellis, J. Quevillon, T. You, and Z. Zhang, Phys. Lett. B 762, 166 (2016).

[46] B. Henning, X. Lu, and H. Murayama, J. High Energy Phys. 01 (2018) 123.

[47] S. A. R. Ellis, J. Quevillon, T. You, and Z. Zhang, J. High Energy Phys. 08 (2017) 054.

[48] B. Summ and A. Voigt, J. High Energy Phys. 08 (2018) 026.

[49] M. Krämer, B. Summ, and A. Voigt, J. High Energy Phys. 01 (2020) 079.

[50] Q.-H. Cao, S.-L. Chen, and Y. Liu, Phys. Rev. D 95, 053004 (2017).

[51] C. Englert, G. F. Giudice, A. Greljo, and M. Mccullough, J. High Energy Phys. 09 (2019) 041.

[52] M. Aaboud et al. (ATLAS Collaboration), Phys. Rev. D 99, 052009 (2019).

[53] A. M. Sirunyan et al. (CMS Collaboration), Eur. Phys. J. C 78, 140 (2018).

[54] A. M. Sirunyan et al. (CMS Collaboration), J. High Energy Phys. 11 (2019) 082.

[55] CMS Collaboration, CERN Report No. CMS-PAS-FTR18-031, 2018, https://cds.cern.ch/record/2650211.

[56] M. Aaboud et al. (ATLAS Collaboration), CERN Tech. Report No. ATL-PHYS-PUB-2018-047, 2018, http://cds .cern.ch/record/2651870.

[57] P. Azzi et al. (HL-LHC and HE-LHC Working Group), CERN Yellow Reports: Monographs (CERN, Geneva,
2019), Vol. 7, pp. 1-220, https://cds.cern.ch/record/ $2650160 / ? \ln =\mathrm{en}$.

[58] C. Arina et al., J. High Energy Phys. 11 (2016) 111.

[59] S. Banerjee, M. Chala, and M. Spannowsky, Eur. Phys. J. C 78, 683 (2018).

[60] M. Czakon, D. Heymes, A. Mitov, D. Pagani, I. Tsinikos, and M. Zaro, J. High Energy Phys. 10 (2017) 186.

[61] E. Vryonidou and C. Zhang, J. High Energy Phys. 08 (2018) 036.

[62] F. Maltoni, L. Mantani, and K. Mimasu, J. High Energy Phys. 10 (2019) 004.

[63] C. Anastasiou, C. Duhr, F. Dulat, F. Herzog, and B. Mistlberger, Phys. Rev. Lett. 114, 212001 (2015).

[64] C. Anastasiou, C. Duhr, F. Dulat, E. Furlan, T. Gehrmann, F. Herzog, A. Lazopoulos, and B. Mistlberger, J. High Energy Phys. 05 (2016) 058.

[65] K. J. F. Gaemers and F. Hoogeveen, Phys. Lett. 146B, 347 (1984).

[66] D. Dicus, A. Stange, and S. Willenbrock, Phys. Lett. B 333, 126 (1994).

[67] W. Bernreuther, A. Brandenburg, and M. Flesch, arXiv: hep-ph/9812387.

[68] R. Frederix and F. Maltoni, J. High Energy Phys. 01 (2009) 047.

[69] V. Barger, T. Han, and D. G. E. Walker, Phys. Rev. Lett. 100, 031801 (2008).

[70] N. Craig, F. D’Eramo, P. Draper, S. Thomas, and H. Zhang, J. High Energy Phys. 06 (2015) 137.

[71] W. Bernreuther, P. Galler, C. Mellein, Z. G. Si, and P. Uwer, Phys. Rev. D 93, 034032 (2016).

[72] B. Hespel, F. Maltoni, and E. Vryonidou, J. High Energy Phys. 10 (2016) 016.

[73] M. Carena and Z. Liu, J. High Energy Phys. 11 (2016) 159.

[74] D. B. Franzosi, F. Fabbri, and S. Schumann, J. High Energy Phys. 03 (2018) 022.

[75] P. Basler, S. Dawson, C. Englert, and M. Mühlleitner, Phys. Rev. D 99, 055048 (2019).

[76] A. Djouadi, J. Ellis, A. Popov, and J. Quevillon, J. High Energy Phys. 03 (2019) 119.

[77] N. Kauer, A. Lind, P. Maierhöfer, and W. Song, J. High Energy Phys. 07 (2019) 108.

[78] J. Brehmer, A. Freitas, D. Lopez-Val, and T. Plehn, Phys. Rev. D 93, 075014 (2016).

[79] D. B. Franzosi and C. Zhang, Phys. Rev. D 91, 114010 (2015).

[80] A. Freitas, D. López-Val, and T. Plehn, Phys. Rev. D 94, 095007 (2016).

[81] D. B. Franzosi, E. Vryonidou, and C. Zhang, J. High Energy Phys. 10 (2017) 096.

[82] K. Arnold et al., Comput. Phys. Commun. 180, 1661 (2009).

[83] J. Baglio et al., arXiv:1107.4038.

[84] K. Arnold et al., arXiv:1207.4975.

[85] J. Baglio et al., arXiv:1404.3940.

[86] T. Hahn, Acta Phys. Pol. B 30, 3469 (1999).

[87] T. Hahn and C. Schappacher, Comput. Phys. Commun. 143, 54 (2002).

[88] J. Alwall, R. Frederix, S. Frixione, V. Hirschi, F. Maltoni, O. Mattelaer, H. S. Shao, T. Stelzer, P. Torrielli, and M. Zaro, J. High Energy Phys. 07 (2014) 079. 
[89] M. Nowakowski and A. Pilaftsis, Z. Phys. C 60, 121 (1993).

[90] M. H. Seymour, Phys. Lett. B 354, 409 (1995).

[91] G. Passarino, C. Sturm, and S. Uccirati, Nucl. Phys. B834, 77 (2010).

[92] N. Kauer and C. O'Brien, Eur. Phys. J. C 75, 374 (2015).

[93] C. Englert, I. Low, and M. Spannowsky, Phys. Rev. D 91, 074029 (2015).

[94] S. Goria, G. Passarino, and D. Rosco, Nucl. Phys. B864, 530 (2012).

[95] R. Contino, A. Falkowski, F. Goertz, C. Grojean, and F. Riva, J. High Energy Phys. 07 (2016) 144.

[96] I. Brivio, S. Bruggisser, F. Maltoni, R. Moutafis, T. Plehn, E. Vryonidou, S. Westhoff, and C. Zhang, arXiv:1910 .03606.

[97] M. Czakon, P. Fiedler, and A. Mitov, Phys. Rev. Lett. 110, 252004 (2013).

[98] M. Czakon, D. Heymes, and A. Mitov, Phys. Rev. Lett. 116, 082003 (2016).
[99] M. Czakon, A. Ferroglia, D. Heymes, A. Mitov, B. D. Pecjak, D. J. Scott, X. Wang, and L. L. Yang, J. High Energy Phys. 05 (2018) 149.

[100] G. Bevilacqua and M. Worek, J. High Energy Phys. 07 (2012) 111.

[101] R. Frederix, D. Pagani, and M. Zaro, J. High Energy Phys. 02 (2018) 031.

[102] N. Greiner, K. Kong, J.-C. Park, S. C. Park, and J.-C. Winter, J. High Energy Phys. 04 (2015) 029.

[103] S. Baek, P. Ko, and P. Wu, J. High Energy Phys. 10 (2016) 117.

[104] P. J. Fox, I. Low, and Y. Zhang, J. High Energy Phys. 03 (2018) 074.

[105] G. Buchalla, A. J. Buras, and M. E. Lautenbacher, Rev. Mod. Phys. 68, 1125 (1996).

[106] A. Denner and S. Dittmaier, Nucl. Phys. B, Proc. Suppl. 157, 53 (2006).

[107] G. Passarino and M. J. G. Veltman, Nucl. Phys. B160, 151 (1979). 